\title{
Exact Bayes' Theorem Based Probabilistic Data Association for Iterative MIMO Detection and Decoding
}

\author{
Shaoshi Yang and Lajos Hanzo \\ School of Electronics and Computer Science, University of Southampton, Southampton, SO17 1BJ, UK \\ Email: $\{$ sy7g09, lh $\} @$ ecs.soton.ac.uk, http://www.cspc.ecs.soton.ac.uk
}

\begin{abstract}
In our previous work, it was shown that the conventional approximate Bayes' theorem based probabilistic data association (PDA) algorithms output "nominal APPs", which are unsuitable for the classic architecture of iterative detection and decoding (IDD) aided receivers. To circumvent this predicament, in this paper we propose an exact Bayes' theorem based logarithmic domain PDA (EB-Log-PDA) method, whose output has similar characteristics to the true APPs, and hence it is readily applicable to the classic IDD architecture of multipleinput multiple-output (MIMO) systems using $M$-ary modulation. Furthermore, we demonstrate that introducing inner iterations into EB-Log-PDA, which is common practice in conventionalPDA aided uncoded MIMO systems, would actually degrade the IDD receiver's performance, despite significantly increasing the overall computational complexity of the IDD receiver. Finally, we show that the EB-Log-PDA based IDD scheme operating without any inner PDA iterations has a similar performance to that of the optimal maximum a posteriori (MAP) detector based IDD receiver, while imposing a significantly lower computational complexity in the scenarios considered.
\end{abstract}

Index Terms-Bayes' theorem, iterative detection and decoding, multiple-input multiple-output (MIMO), probabilistic data association (PDA), $M$-ary modulation.

\section{INTRODUCTION}

The probabilistic data association (PDA) approach has been recently invoked for low-complexity, high-performance softinput soft-output (SISO) detection/equalization in multipleinput multiple-output (MIMO) fading channels [1]-[6]. In this scenario, the probabilities of the potential candidate symbols serve as the soft input/output information and are conventionally estimated using an approximate version of the Bayes' theorem relying on a self-iterative process.

These symbol probabilities have historically been interpreted as a posteriori probabilities (APPs) without causing any notable problems. This is mainly because most contributions concerning the PDA algorithm were based on uncoded systems, where the PDA algorithm's applicability does not require an accurate interpretation of the rigorous mathematical nature of its output symbol probabilities, and in particular, the calculation of the bit-wise extrinsic log-likelihood ratios (LLRs) is not required [1]-[6]. In our recent work [7], we pointed out, however, that this conventional wisdom is not

The financial support of the China Scholarship Council (CSC), of the Research Councils UK (RCUK) under the India-UK Advanced Technology Center (IU-ATC), of the EU under the auspices of the Concerto project, and of the European Research Councils Advanced Fellow Grant is gratefully acknowledged. directly applicable in iterative receivers. More explicitly, we argued that the output symbol probabilities of all approximate Bayes' theorem based conventional PDA detectors/equalizers constitute "nominal APPs" rather than true APPs. Due to this misconception, the problem of designing a powerful PDA-based iterative detection and decoding (IDD) receiver became challenging. As a remedy, in [7] we first answered the question of how to generate the bit-wise extrinsic LLRs using the symbol-wise "nominal APPs", and then proposed an IDD receiver employing the approximate Bayes' theorem based logarithmic-domain PDA (AB-Log-PDA) for $M$-ary modulation aided MIMO systems.

The AB-Log-PDA based IDD receiver of [7] resulted in an irregular IDD architecture, where the bit-wise extrinsic LLRs output by the AB-Log-PDA detector cannot be generated by subtracting the bit-wise a priori LLRs from the bit-wise $a$ posteriori LLRs. In other words, the canonical relationship of $\mathbf{L}_{E}=\mathbf{L}_{D}-\mathbf{L}_{A}$ no longer holds for conventional approximate Bayes' theorem based PDA detectors. On the other hand, the classic IDD architecture has been widely recognized, whose constituent soft detector is typically expected to satisfy $\mathbf{L}_{E}=$ $\mathbf{L}_{D}-\mathbf{L}_{A}$. Therefore, in contrast to the existing PDAs [1]-[7] all of which output "nominal APPs", it might be promising to develop a PDA-based algorithm capable of generating the true APPs for facilitating the employment of PDA in classic IDD architectures.

In this paper we propose an exact Bayes' theorem based logarithmic-domain PDA (EB-Log-PDA) detector for the IDD of forward error correction (FEC)-coded MIMO systems using arbitrary $M$-ary modulation. As opposed to the existing PDAs relying on an approximate version of the Bayes' theorem [1]-[7], the proposed EB-Log-PDA's output exhibits similar characteristics to the true APPs and thus $\mathbf{L}_{E}=\mathbf{L}_{D}-\mathbf{L}_{A}$ can be satisfied. As a benefit, the proposed EB-Log-PDA approach becomes immediately applicable to the classic IDD architecture. Furthermore, we investigate the impact of the inner iterations within the EB-Log-PDA algorithm on the achievable performance of the corresponding IDD scheme. We demonstrate that, similar to the case of AB-Log-PDA, the performance of the proposed EB-Log-PDA based IDD scheme is consistently degraded as the number of inner iterations within the EB-Log-PDA increases. This is in stark contrast to PDA detectors invoked in uncoded MIMO systems. Therefore, we conclude that as far as IDD receivers are concerned, both 


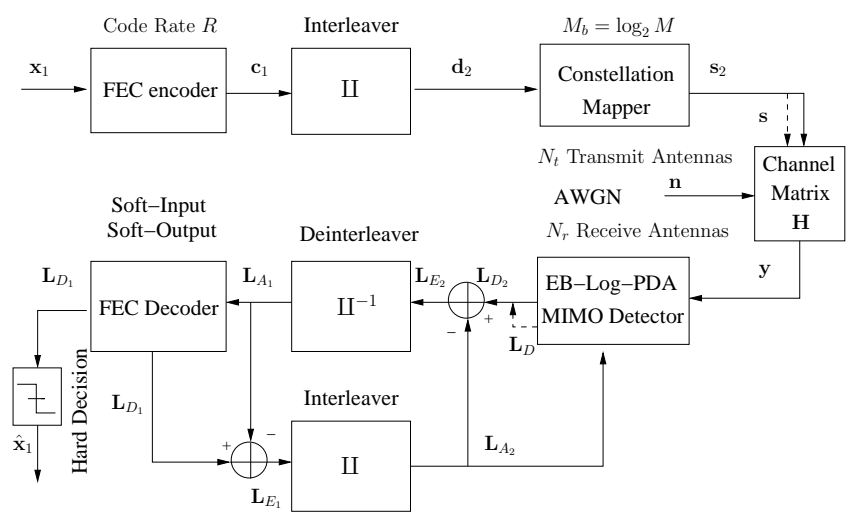

Fig. 1. FEC-coded MIMO system with PDA methods based iterative detection and decoding receiver. The subscript " 1 " denotes the processing modules associated with the "outer" FEC encoder/decoder, and the subscript "2" denotes the processing modules that are connected with the "inner" spacetime mapper/detector. The arrow with dashed line indicates that $\mathbf{s}$ and $\mathbf{L}_{D}$ are the subvectors of $\mathbf{s}_{2}$ and $\mathbf{L}_{D_{2}}$, respectively.

the EB-Log-PDA detector and the AB-Log-PDA detector [7] should dispense with their inner iterations. Finally, we show that the resultant EB-Log-PDA based IDD receiver dispensing with inner PDA iterations exhibits a performance close to that of the optimal maximum a posteriori (MAP)-based IDD scheme, while imposing a significantly lower computational complexity in the scenarios considered. A more comprehensive investigation of the relationship between the AB-Log-PDA and the EB-Log-PDA based IDDs is provided in [8].

\section{SYSTEM MODEL}

Similar to [7], we consider the FEC-coded spatial multiplexing MIMO system of Fig. 1. At the output of the fading channel $\mathbf{H}$, the received $\left(N_{r} \times 1\right)$-element complex-valued baseband signal vector per channel use is represented by

$$
\mathbf{y}=\mathbf{H s}+\mathbf{n},
$$

where $\mathbf{s}=\left[s_{1}, s_{2}, \cdots, s_{N_{t}}\right]^{T}$ is normalized by the componentwise energy constraint $\mathbb{E}\left(\left|s_{i}\right|^{2}\right)=E_{\mathbf{s}} / N_{t}$ in order to ensure a total transmit energy of $E_{\mathbf{s}}$ per channel use, and $\mathbf{s}$ does not contain any additional space-time coding; each symbol $s_{i}$ is taken from a modulation constellation $\mathcal{A}=\left\{a_{1}, a_{2}, \cdots, a_{M}\right\}$, where $M_{b}=\log _{2} M$ is the number of bits per symbol; $\mathbf{n}$ is the $\left(N_{r} \times 1\right)$-element zero-mean complex-valued circularly symmetric Gaussian noise vector having a covariance matrix of $2 \sigma^{2} \mathbf{I}_{N_{r}}$, where $\mathbf{I}_{N_{r}}$ represents an $\left(N_{r} \times N_{r}\right)$-element identity matrix; and $\mathbf{H}$ is an $\left(N_{r} \times N_{t}\right)$-element complexvalued matrix with entries of $h_{j i}$, which are perfectly known to the receiver, $j=1, \cdots, N_{r}, i=1, \cdots, N_{t}, N_{r} \geq N_{t}$. Similar to [7], in this paper we also assume that $h_{j i}$ is subject to uncorrelated Nakagami- $m$ fading.

\section{The EB-Log-PDA ALgorithm FOR IDD RECEIVER}

In contrast to the AB-Log-PDA proposed in [7], where the so-called non-decoupled signal model was used, below we use the decoupled signal model in order to further reduce the computational complexity for the scenario of $N_{r}>N_{t}$ (see the complexity comparison results presented in Section V-B for more details). When relying on the zero-forcing principle based preprocessing, the received signal model of (1) may be rewritten as

$$
\tilde{\mathbf{y}}=\mathbf{s}+\tilde{\mathbf{n}}=s_{i} \mathbf{e}_{i}+\underbrace{\sum_{k \neq i} s_{k} \mathbf{e}_{k}}_{\mathbf{u}_{i}}+\tilde{\mathbf{n}} \triangleq s_{i} \mathbf{e}_{i}+\underbrace{\mathbf{u}_{i}+\tilde{\mathbf{n}}}_{\mathbf{v}_{i}},
$$

where we have $\tilde{\mathbf{y}}=\left(\mathbf{H}^{H} \mathbf{H}\right)^{-1} \mathbf{H}^{H} \mathbf{y}, \tilde{\mathbf{n}}=\left(\mathbf{H}^{H} \mathbf{H}\right)^{-1} \mathbf{H}^{H} \mathbf{n}$, and $\mathbf{e}_{i}$ denotes an $\left(N_{t} \times 1\right)$-element vector, whose $i$ th element is equal to one and the other elements are equal to zero, $i$, $k=1,2, \cdots, N_{t}$.

For uncoded MIMO systems, where no outer source of a priori soft information about the transmitted symbols is available, the existing PDA methods typically use the received signal $\mathbf{y}$ and the channel matrix $\mathbf{H}$ as input quantities, and then generate the estimated symbol-wise nominal APPs of the transmitted symbols $\left\{s_{i}\right\}_{i=1, \cdots, N_{t}}$ as its output. By contrast, for FEC-coded MIMO systems, we have an extra input quantity, which is the soft information feedback gleaned from the outer FEC decoder. In this scenario, because the reliability of the FEC decoder's output is typically higher than that of the soft MIMO detector at the previous stage, some of the key operations of the proposed EB-Log-PDA are implemented in the logarithmic domain in order to improve the achievable numerical stability and accuracy.

Although the interference-plus-noise term $\mathbf{v}_{i}$ obeys a multimodal Gaussian mixture distribution [7], initially it is plausible to obtain a coarse estimate of $s_{i}$ by assuming that $\mathbf{v}_{i}$ obeys a single $N_{t}$-variate Gaussian distribution. It is worth noting that in the circumstances considered each element of $\mathbf{v}_{i}$ is the sum of only two scalar random variables for any $N_{t} \geq 2$, hence, according to the central limit theorem, the Gaussian approximation of $\mathbf{v}_{i}$ does not become more accurate when $N_{t}$ is increased. This trend is different from that of the nondecoupled signal model based PDA [7]. In order to fully characterize the complex random vector $\mathbf{v}_{i}$ which is not necessarily proper [9], [10], we specify the mean of

$$
\boldsymbol{\mu}_{i} \triangleq \mathbb{E}\left(\mathbf{v}_{i}\right)=\sum_{k \neq i} \mathbb{E}\left(s_{k}\right) \mathbf{e}_{k},
$$

the covariance of

$$
\mathbf{\Upsilon}_{i} \triangleq \mathbb{C}\left(\mathbf{v}_{i}\right)=\sum_{k \neq i} \mathbb{C}\left(s_{k}\right) \mathbf{e}_{k} \mathbf{e}_{k}^{T}+2 \sigma^{2}\left(\mathbf{H}^{H} \mathbf{H}\right)^{-1},
$$

and the pseudo-covariance of

$$
\underline{\Upsilon}_{i} \triangleq \mathbb{C}_{p}\left(\mathbf{v}_{i}\right)=\sum_{k \neq i} \mathbb{C}_{p}\left(s_{k}\right) \mathbf{e}_{k} \mathbf{e}_{k}^{T},
$$

where the pseudo-covariance of a complex random vector $\mathbf{x}$ is defined as [9], [10]

$$
\mathbb{C}_{p}(\mathbf{x}) \triangleq \mathbb{E}\left[(\mathbf{x}-\mathbb{E}(\mathbf{x}))(\mathbf{x}-\mathbb{E}(\mathbf{x}))^{T}\right] .
$$

Note that (5) holds, because $\tilde{\mathbf{n}}$ is a circularly symmetric complex-valued Gaussian noise vector and hence it is proper [9], [10].

Considering the IDD architecture, we define an $\left(N_{t} \times M\right)$ element probability matrix $\mathbf{P}^{\left(z, z^{\prime}\right)}$, whose element $P_{n, m}^{\left(z, z^{\prime}\right)}$ represents the estimate of the APP that we have $s_{n}=a_{m}$ at the 
$z$-th $/ z^{\prime}$-th outer/inner iteration of the EB-Log-PDA approach. More precisely, we have

$$
P_{n, m}^{\left(z, z^{\prime}\right)}=P_{m}^{\left(z, z^{\prime}\right)}\left(s_{n} \mid \tilde{\mathbf{y}}\right) \triangleq P^{\left(z, z^{\prime}\right)}\left(s_{n}=a_{m} \mid \tilde{\mathbf{y}}\right),
$$

where $z$ and $z^{\prime}$ are nonnegative integers, $n=1, \cdots, N_{t}$ and $m=1, \cdots, M$. Then we have

$$
\begin{gathered}
\mathbb{E}\left(s_{k}\right)=\sum_{m=1}^{M} a_{m} P_{m}^{\left(z, z^{\prime}\right)}\left(s_{k} \mid \tilde{\mathbf{y}}\right), \\
\mathbb{C}\left(s_{k}\right)=\sum_{m=1}^{M}\left(a_{m}-\mathbb{E}\left(s_{k}\right)\right)\left(a_{m}-\mathbb{E}\left(s_{k}\right)\right)^{*} P_{m}^{\left(z, z^{\prime}\right)}\left(s_{k} \mid \tilde{\mathbf{y}}\right),
\end{gathered}
$$

and

$$
\mathbb{C}_{p}\left(s_{k}\right)=\sum_{m=1}^{M}\left(a_{m}-\mathbb{E}\left(s_{k}\right)\right)^{2} P_{m}^{\left(z, z^{\prime}\right)}\left(s_{k} \mid \tilde{\mathbf{y}}\right),
$$

for (3), (4) and (5), respectively.

Note that from Eq. (3) to Eq. (10) we effectively use $\left(N_{t}-1\right)$ probability vectors of $\left\{\mathbf{P}^{\left(z, z^{\prime}\right)}(k,:)\right\}_{k \neq i}$ associated with the interference signal $\left\{s_{k}\right\}_{k \neq i}$ to model $\mathbf{v}_{i}$, where $\mathbf{P}^{\left(z, z^{\prime}\right)}(k,:)$ represents the $k$ th row of the matrix $\mathbf{P}^{\left(z, z^{\prime}\right)}$. Since we do not have any outer a priori knowledge about the distribution of $s_{n} \mid \tilde{\mathbf{y}}$ at the beginning, $P_{m}^{\left(z, z^{\prime}\right)}\left(s_{n} \mid \tilde{\mathbf{y}}\right)$ is initialized using the uniform distribution of

$$
P_{m}^{(0,0)}\left(s_{n} \mid \tilde{\mathbf{y}}\right)=\frac{1}{M},
$$

for $\forall n=1, \cdots, N_{t}$ and $\forall m=1, \cdots, M$.

Based on the assumption that $\mathbf{v}_{i}$ obeys the Gaussian distribution, $\tilde{\mathbf{y}} \mid s_{i}$ is also Gaussian distributed. We define

$$
\mathbf{w} \triangleq \tilde{\mathbf{y}}-s_{i} \mathbf{e}_{i}-\sum_{k \neq i} \mathbb{E}\left(s_{k}\right) \mathbf{e}_{k}
$$

and

$$
\alpha_{i, m}^{\left(z, z^{\prime}+1\right)} \triangleq-\left[\begin{array}{c}
\Re(\mathbf{w}) \\
\Im(\mathbf{w})
\end{array}\right]^{T} \mathbf{\Lambda}_{i}^{-1}\left[\begin{array}{c}
\Re(\mathbf{w}) \\
\Im(\mathbf{w})
\end{array}\right],
$$

where the composite covariance matrix $\boldsymbol{\Lambda}_{i}$ is defined as

$$
\mathbf{\Lambda}_{i} \triangleq\left[\begin{array}{cc}
\Re\left(\mathbf{\Upsilon}_{i}+\underline{\mathbf{\Upsilon}}_{i}\right) & -\Im\left(\mathbf{\Upsilon}_{i}-\underline{\mathbf{\Upsilon}}_{i}\right) \\
\Im\left(\mathbf{\Upsilon}_{i}+\underline{\mathbf{\Upsilon}}_{i}\right) & \Re\left(\mathbf{\Upsilon}_{i}-\underline{\mathbf{\Upsilon}}_{i}\right)
\end{array}\right],
$$

where $\Re(\cdot)$ and $\Im(\cdot)$ represent the real and imaginary part of a complex variable, respectively. Then the likelihood function of $\tilde{\mathbf{y}} \mid s_{i}=a_{m}$ at the $\left(z, z^{\prime}+1\right)$-st iteration satisfies

$$
p_{m}^{\left(z, z^{\prime}+1\right)}\left(\tilde{\mathbf{y}} \mid s_{i}\right) \propto \exp \left(\alpha_{i, m}^{\left(z, z^{\prime}+1\right)}\right) .
$$

In the next step, the existing PDA methods employed in uncoded MIMO systems typically invoked an approximate form of the Bayes' theorem to estimate the symbol APPs [1] [7], which is

$$
\mathcal{P}_{m}^{\left(z, z^{\prime}+1\right)}\left(s_{i} \mid \tilde{\mathbf{y}}\right) \approx \frac{p_{m}^{\left(z, z^{\prime}+1\right)}\left(\tilde{\mathbf{y}} \mid s_{i}\right)}{\sum_{m=1}^{M} p_{m}^{\left(z, z^{\prime}+1\right)}\left(\tilde{\mathbf{y}} \mid s_{i}\right)} .
$$

However, we argue that (16) does not conform to the formal mathematical definition of the APP in Bayes' statistics, ${ }^{1}$ which is [11]

$$
P(\theta \mid X) \propto P(\theta) P(X \mid \theta),
$$

where the probability $P(\theta)$ is an a priori belief of the random variable $\theta$, and $P(X \mid \theta)$ is the likelihood of the observation $X$. Therefore, $\mathcal{P}_{m}^{\left(z, z^{\prime}+1\right)}\left(s_{i} \mid \tilde{\mathbf{y}}\right)$ calculated with the aid of (16) does not represent the true APPs, but instead the normalized likelihoods, which may be regarded as a type of nominal APPs.

To obtain the true APP of symbol $s_{i}$ at the $\left(z, z^{\prime}+1\right)$-st iteration of the PDA-aided IDD receiver, we advocate to use the exact form of the Bayes' theorem. Hence the true APP estimated at the output of the PDA method is given as

$$
\begin{aligned}
& P_{m}^{\left(z, z^{\prime}+1\right)}\left(s_{i} \mid \tilde{\mathbf{y}}\right) \\
= & \frac{p_{m}^{\left(z, z^{\prime}+1\right)}\left(\tilde{\mathbf{y}} \mid s_{i}\right) P_{m}^{\left(z, z^{\prime}\right)}\left(s_{i}\right)}{\sum_{m=1}^{M} p_{m}^{\left(z, z^{\prime}+1\right)}\left(\tilde{\mathbf{y}} \mid s_{i}\right) P_{m}^{\left(z, z^{\prime}\right)}\left(s_{i}\right)} \\
= & \frac{\exp \left(\beta_{i, m}^{\left(z, z^{\prime}+1\right)}-\gamma\right)}{\sum_{m=1}^{M} \exp \left(\beta_{i, m}^{\left(z, z^{\prime}+1\right)}-\gamma\right)},
\end{aligned}
$$

where $P_{m}^{\left(z, z^{\prime}\right)}\left(s_{i}\right)$ is the a priori probability generated from the extrinsic LLRs fed back by the soft FEC decoder, and $\beta_{i, m}^{\left(z, z^{\prime}+1\right)} \triangleq \alpha_{i, m}^{\left(z, z^{\prime}+1\right)}+\ln \left(P_{m}^{\left(z, z^{\prime}\right)}\left(s_{i}\right)\right)$. Note that $\gamma \triangleq$ $\max _{m=1, \cdots, M} \beta_{i, m}^{\left(z, z^{\prime}+1\right)}$ is introduced for further improving the numerical stability and accuracy. For the same reason, (18) is reformulated in the logarithmic domain as

$$
\begin{aligned}
& \psi_{i, m}^{\left(z, z^{\prime}+1\right)} \\
\triangleq & \ln \left(P_{m}^{\left(z, z^{\prime}+1\right)}\left(s_{i} \mid \tilde{\mathbf{y}}\right)\right) \\
= & \tilde{\beta}_{i, m}^{\left(z, z^{\prime}+1\right)}-\ln \left(\sum_{m=1}^{M} \exp \left(\tilde{\beta}_{i, m}^{\left(z, z^{\prime}+1\right)}\right)\right),
\end{aligned}
$$

where we have $\tilde{\beta}_{i, m}^{\left(z, z^{\prime}+1\right)} \triangleq \beta_{i, m}^{\left(z, z^{\prime}+1\right)}-\gamma$, and the second term of the right-hand-side expression may be computed by invoking the "Jacobian logarithm' of [12]. When invoking the Max-log approximation, (19) may be further simplified as

$$
\psi_{i, m}^{\left(z, z^{\prime}+1\right)} \approx \tilde{\beta}_{i, m}^{\left(z, z^{\prime}+1\right)}-\max _{m=1, \cdots, M} \tilde{\beta}_{i, m}^{\left(z, z^{\prime}+1\right)}=\tilde{\beta}_{i, m}^{\left(z, z^{\prime}+1\right)} .
$$

As a result, the estimated symbol APP of $s_{i}$ is given by

$$
P_{m}^{\left(z, z^{\prime}+1\right)}\left(s_{i} \mid \tilde{\mathbf{y}}\right) \approx e^{\psi_{i, m}^{\left(z, z^{\prime}+1\right)}},
$$

which will be used for replacing the corresponding element $P_{m}^{\left(z, z^{\prime}\right)}\left(s_{i} \mid \tilde{\mathbf{y}}\right)$ in the probability matrix $\mathbf{P}^{\left(z, z^{\prime}\right)}$. Based on these updated symbol APPs, the procedure presented above may be repeated either in the next inner iteration within the PDA or in the next outer iteration exchanging extrinsic information between the FEC-decoder and the MIMO detector to obtain new estimates of the symbol APPs.

For the sake of clarity, the EB-Log-PDA algorithm relying

${ }^{1}$ For the sake of clarity, here we use $\mathcal{P}()$ to denote the symbol probabilities estimated using the approximate Bayes' formula given by (16), while using $P()$ to represent ordinary probabilities otherwise. 
TABLE I

SUMMARY OF THE EB-LOG-PDA ALGORITHM

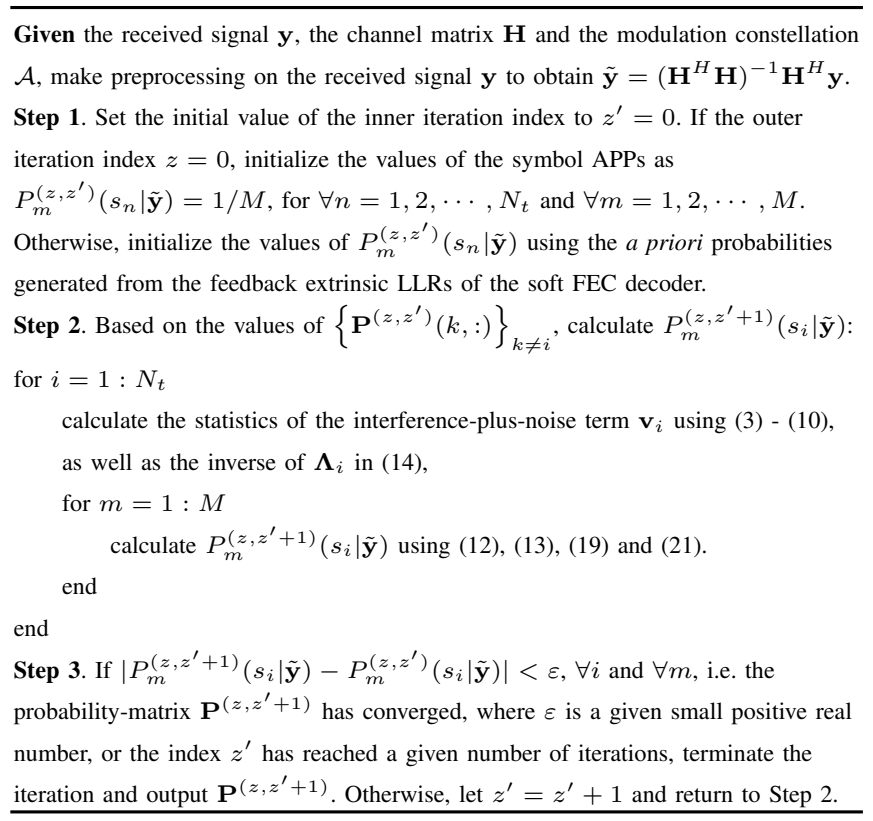

on the a priori soft information feedback gleaned from the outer FEC decoder is summarized in Table I.

\section{Extrinsic LLR CALCULATION For EB-Log-PDA}

For ease of exposition, in the following we will denote the left-hand-side term of (18) as $P\left(s_{i}=a_{m} \mid \mathbf{y}\right)$. Proposition 1 of [7] demonstrated that the classic candidate-search based approach of calculating the extrinsic LLRs [12] is not applicable to the family of PDA algorithms. However, as a beneficial result of replacing (16) by (18), the extrinsic LLRs may be calculated according to the canonical relationship by subtracting the a priori LLRs from the a posteriori LLRs that are generated from the estimated symbol APPs of the EB-LogPDA detector, hence we have

$$
\begin{aligned}
L_{E}^{E B}\left(b_{i l} \mid \mathbf{y}\right)= & L_{D}\left(b_{i l} \mid \mathbf{y}\right)-L_{A}\left(b_{i l}\right) \\
= & \ln \frac{P\left(b_{i l}=+1 \mid \mathbf{y}\right)}{P\left(b_{i l}=-1 \mid \mathbf{y}\right)}-L_{A}\left(b_{i l}\right) \\
= & \ln \frac{\sum_{\forall a_{m} \in \mathcal{A}_{l}^{+}} P\left(s_{i}=a_{m} \mid \mathbf{y}\right)}{\sum_{\forall a_{m} \in \mathcal{A}_{l}^{-}} P\left(s_{i}=a_{m} \mid \mathbf{y}\right)} \\
& -\underbrace{\ln \frac{P\left(b_{i l}=+1\right)}{P\left(b_{i l}=-1\right)}}_{L_{A}\left(b_{i l}\right)},
\end{aligned}
$$

where $\mathcal{A}_{l}^{ \pm}$denotes the set of $M / 2$ constellation points whose $l$ th bit is +1 or -1 , respectively. It is noteworthy that compared to the candidate-search based method, (22) represents a simpler technique of generating the bit-wise extrinsic LLRs $L_{E}^{E B}\left(b_{i l} \mid \mathbf{y}\right)$, as long as the true symbol APPs of $P\left(s_{i}=a_{m} \mid \mathbf{y}\right)$ may be obtained.

Unfortunately, this approach is not applicable to the conventional approximate Bayes' theorem based PDA methods [1]-[7]. Our study reveals that the estimated symbol-wise

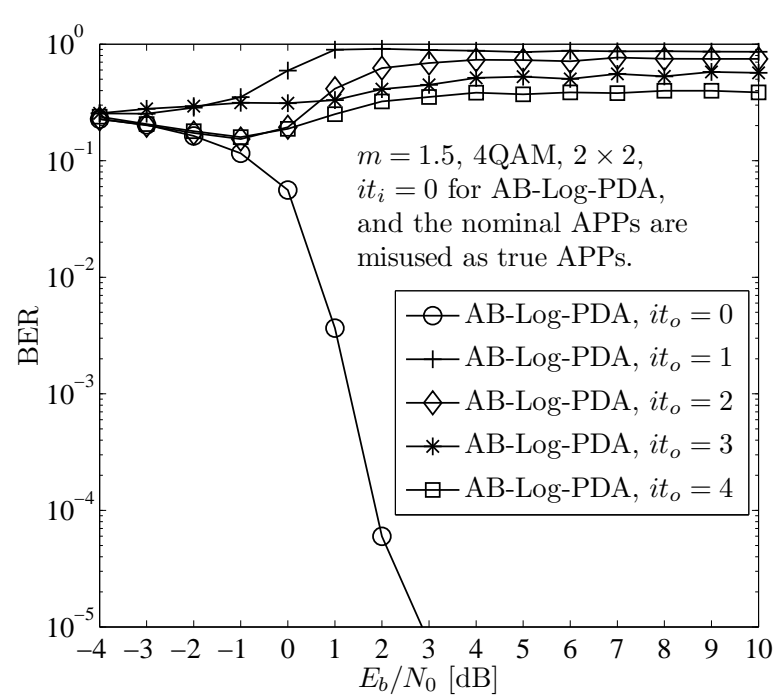

Fig. 2. BER of the AB-Log-PDA based IDD scheme, where the nominal symbol APPs were misinterpreted as the true symbol APPs.

nominal APPs obtained from (16) are unsuitable for generating the correct bit-wise extrinsic LLRs upon invoking the classic formula (22). This hidden fact is corroborated by the simulation results of Fig. 2. In the scenarios where the number of outer iterations $i t_{o}$ was set to be higher than zero, it was observed in Fig. 2 that the BER results of the IDD scheme using the AB-Log-PDA and (22) became abnormally poor, when the symbol-wise nominal APPs produced by (16) were misinterpreted as symbol-wise true APPs. More specifically, we can observe from Fig. 2 that except for $i t_{o}=0$, the BER of the AB-Log-PDA based IDD scheme unexpectedly degrades upon increasing $E_{b} / N_{0}$, and it also deteriorates when $i t_{o}$ increases from 1 to 4 . The BER curve of the $i t_{o}=0$ scenario characterized in Fig. 2 exhibits a trend in line with our expectations, because in this case no soft information is fed back from the FEC decoder and hence the term $P_{m}^{\left(z, z^{\prime}\right)}\left(s_{i}\right)$ in (18) can be eliminated. In other words, (16) becomes equivalent to (18) in this scenario. These results further demonstrate that (16) should be regarded as a sort of symbol-wise nominal APP, rather than the symbol-wise true APP, as calculated in (18). As a result, we argue that in order to generate the correct symbol APPs, which are readily applicable to (22), the calculation of $P_{m}^{\left(z, z^{\prime}+1\right)}\left(s_{i} \mid \tilde{\mathbf{y}}\right)$ has to rely on (18) instead of (16) in the PDA based MIMO detector.

\section{Simulation Results and Discussions}

In this section, we characterize the convergence behavior and the achievable performance of the proposed EB-LogPDA based IDD scheme with the aid of both the semianalytical extrinsic information transfer (EXIT) charts [13] and Monte-Carlo simulations. Furthermore, we investigate the impact of inner PDA iterations on the attainable performance of the EB-Log-PDA based IDD scheme. Additionally, the complexity of the proposed EB-Log-PDA based IDD scheme is compared with that of both the AB-Log-PDA and the optimal MAP based IDD schemes, which demonstrates the 


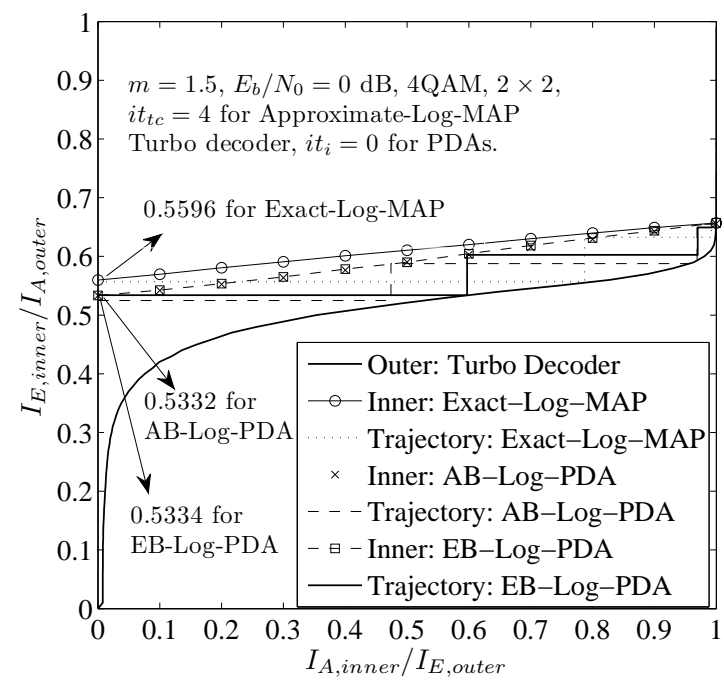

Fig. 3. EXIT chart analysis of the EB-Log-PDA, AB-Log-PDA and ExactLog-MAP based IDD schemes.

attractive performance versus complexity tradeoff achieved by the proposed EB-Log-PDA based IDD scheme.

The turbo code employed uses two constituent recursive systematic convolutional (RSC) codes concatenated in parallel [14]. The RSC codes have a constraint length of $L=3$ and generator polynomials of $(7,5)$ expressed in octal form, where half of the parity bits generated by each of the two RSC codes are punctured, so that the turbo code employed has a coding rate of $R=\frac{k}{n}=1 / 2$. The turbo code is decoded by the Approximate-Log-MAP algorithm using $i t_{t c}=4$ inner iterations. The interleaver employed is a 2400-bit random sequence interleaver. The remaining scenario-dependent simulation parameters are shown in the respective figures, where the MIMO arrangement is represented as $\left(N_{t} \times N_{r}\right)$.

\section{A. Convergence and Performance of EB-Log-PDA based IDD}

Fig. 3 compares the convergence behavior of both the proposed EB-Log-PDA based IDD, as well as of the AB-LogPDA based IDD and of the optimal Exact-Log-MAP based IDD schemes using EXIT chart [13] analysis. It is observed that the EXIT curve of the EB-Log-PDA is close to that of the Exact-Log-MAP, and almost overlaps with that of the AB-LogPDA. More particularly, when the a priori mutual information (MI) is $I_{A \text {,inner }}=0$, the Exact-Log-MAP has the highest extrinsic MI of $I_{E \text {,outer }}=0.5596$, while the EB-Log-PDA has a higher extrinsic MI than the the AB-Log-PDA, which is $I_{E, \text { outer }}=0.5334$ versus $I_{E, \text { outer }}=0.5332$. This indicates that the achievable performances of the EB-Log-PDA and of the AB-Log-PDA are similar to each other, and both of them are close to that of the optimal Exact-Log-MAP in the scenario considered. Additionally, the Monte-Carlo simulation based detection/decoding trajectories indicate that all the three IDD schemes converge after three iterations. Furthermore, the performance improvements achieved at each iteration by the EB-Log-PDA are more significant than those of the AB-LogPDA, but less significant than those of the Exact-Log-MAP.

The above EXIT chart based performance predictions and

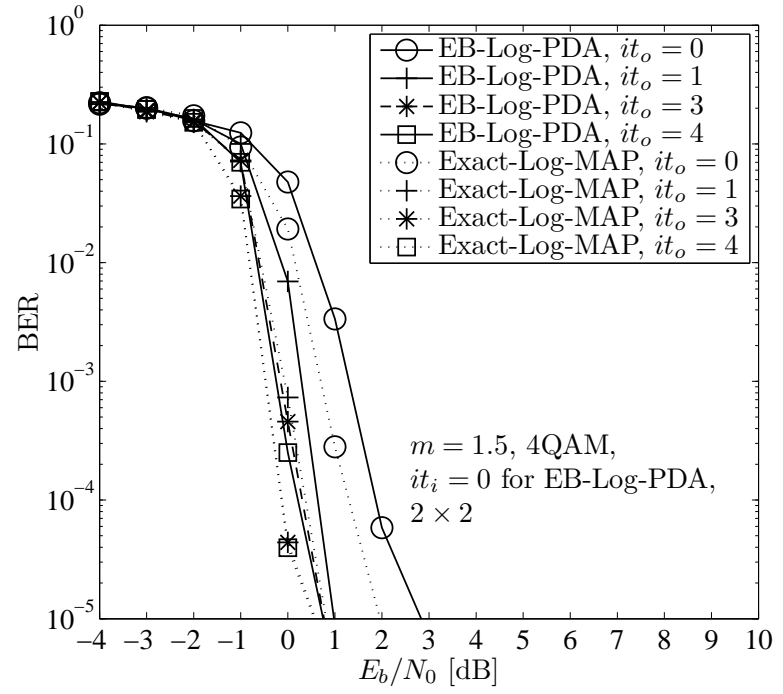

Fig. 4. Impact of the number of outer iterations on BER of the EB-Log-PDA based IDD scheme.

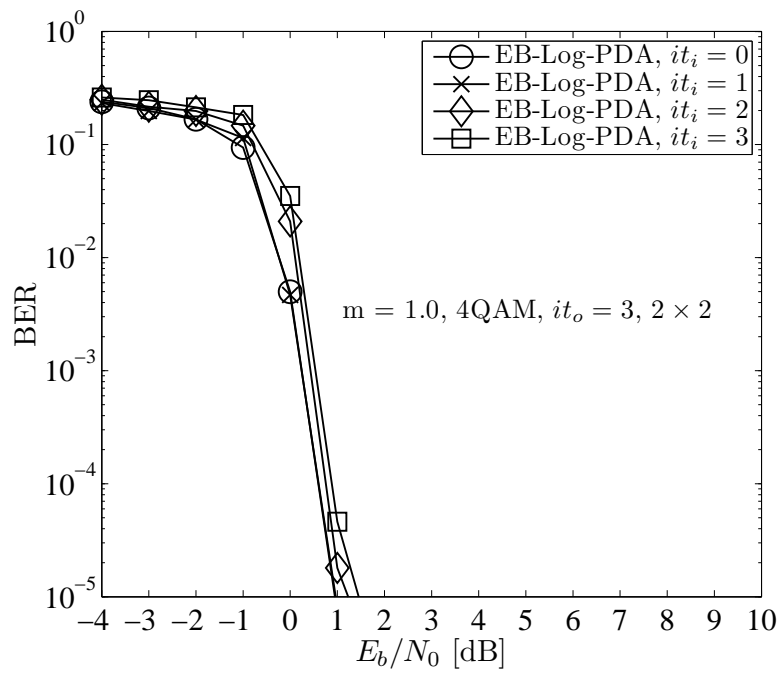

Fig. 5. Impact of the number of inner iterations within the EB-Log-PDA on BER of the EB-Log-PDA based IDD scheme.

the convergence behavior of the IDD schemes considered are also characterized in terms of the BER performance results of Fig. 4, where the Nakagami- $m$ fading parameter is set to $m=1.5$. Observe from Fig. 4 that the performance of the EBLog-PDA based IDD scheme is improved upon increasing the number of outer iterations $i t_{o}$, where $i t_{o}=0$ represents the conventional receiver structure in which the MIMO detector and the FEC decoder are serially concatenated, but operate without exchanging soft information. However, the attainable improvements become gradually smaller and the performance achieved after three outer iterations in Fig. 4 becomes similar to that of four outer iterations. This implies that the EB-LogPDA based IDD scheme essentially converges after three outer iterations. A similar convergence profile is also observed in Fig. 4 for the optimal Exact-Log-MAP based IDD, although its performance is always marginally better than that of the 
corresponding EB-Log-PDA based IDD.

In Fig. 5, we investigate the impact of the number of inner iterations of the EB-Log-PDA on the achievable performance of the IDD scheme. We observe that the performance of the IDD scheme is degraded as the number of its inner iterations increases, despite its increased computational complexity. This implies that the optimal number of inner iterations of the EBLog-PDA conceived for the IDD receiver is $i t_{i}=z^{\prime}=0$. This is because the inner PDA iterations typically fail to assist the iterative Gaussian approximation process in finding the global optimum [5]. Hence, when the reliability of the soft information fed back from the FEC decoder is beyond the reliability limit that the inner iteration aided PDA can achieve, the better-quality feedback of the FEC decoder tends to be degraded towards this limit. Therefore, we conclude that it is not recommended to incorporate inner iterations into the EBLog-PDA algorithm, when designing the EB-Log-PDA IDD scheme.

\section{B. Complexity Comparison}

The computational complexity of the proposed EB-LogPDA based IDD scheme may be evaluated by simply comparing its complexity to those of the AB-Log-PDA and the Exact-Log-MAP based IDDs in a single (outer) iteration. This is because 1) the turbo codec module is common to all the IDD schemes considered; 2) we have shown that the EB-Log-PDA, as well as the AB-Log-PDA and the Exact-Log-MAP based IDD schemes all converge after three outer iterations in the scenarios considered; 3) the PDAs' inner iterations should not be invoked when designing IDD schemes. As shown in Table I, the major computational cost of the EB-Log-PDA per transmit symbol is the calculation of $\boldsymbol{\Lambda}_{i}^{-1}$ and the matrix multiplication of (13). By using the Sherman-Morrison-Woodbury formula based complexity-reduction techniques of [1], the computational cost of calculating $\Lambda_{i}^{-1}$ can be reduced to $\mathcal{O}\left(4 N_{t}^{3}\right)$ real operations per iteration. Additionally, the calculation of (13) requires $\mathcal{O}\left(4 M N_{t}^{3}+2 M N_{t}^{2}\right)$ real operations per iteration. In summary, in terms of real operations, the computational complexity of the decoupled signal model based EB-Log-PDA method is $\mathcal{O}\left(4 M N_{t}^{3}+2 M N_{t}^{2}\right)+\mathcal{O}\left(4 N_{t}^{3}\right)$ per outer iteration, which is not related to the number of receive antennas $N_{r}$, as opposed to the IDD scheme using the non-decoupled signal model based AB-Log-PDA, which has a computational complexity of $\mathcal{O}\left(4 M N_{t} N_{r}^{2}+2 M N_{t} N_{r}\right)+\mathcal{O}\left(4 N_{t} N_{r}^{2}\right)$ per outer iteration [7]. This implies that the decoupled model based EB-Log-PDA has a lower computational complexity than the non-decoupled model based AB-Log-PDA in the scenario of $N_{r}>N_{t}$, which is particularly important, because $N_{r}>N_{t}$ is a typical configuration for spatial multiplexing based MIMO systems.

By comparison, the Exact-Log-MAP algorithm has to calculate the Euclidean distance of $\|\mathbf{y}-\mathbf{H s}\|^{2} M^{N_{t}}$ times per bit per outer iteration [12], hence its complexity order is $\mathcal{O}\left(M^{N_{t}} N_{t} \log _{2} M\right)$ per outer iteration. More specifically, the evaluation of $\|\mathbf{y}-\mathbf{H s}\|^{2}$ requires $\mathcal{O}\left(4 N_{r} N_{t}+6 N_{r}\right)$ real operations. Therefore, the Exact-Log-MAP algorithm has an exponentially increasing computational complexity of $\mathcal{O}\left[M^{N_{t}} N_{t} \log _{2} M\left(4 N_{r} N_{t}+6 N_{r}\right)\right]$ real operations per outer iteration, which is significantly higher than that of the EBLog-PDA, especially when $N_{t}, N_{r}$ and $M$ have large values.

\section{CONCLUSIONS}

In contrast with the existing approximate Bayes' theorem based PDA algorithms, we showed in this paper that the estimated symbol probabilities at the output of the proposed EB-Log-PDA exhibit similar characteristics to the true APPs, hence they are readily applicable to the classic IDD architecture of $M$-ary modulation aided MIMO systems. Additionally, we demonstrated that, similar to the case of the AB-Log-PDA proposed in our previous work [7], introducing inner iterations within the EB-Log-PDA also degrades the IDD receiver's performance despite significantly increasing the overall computational complexity of the IDD receiver, which implies that the optimal number of inner iterations of the EB-Log-PDA is zero as well when it is invoked in IDD receivers. Finally, the IDD scheme based on the proposed EB-Log-PDA using no inner PDA iterations is shown to achieve a comparable performance to that of the optimal MAP detector based IDD receiver, while imposing a significantly lower computational complexity in the scenarios considered.

\section{REFERENCES}

[1] J. Luo, K. R. Pattipati, P. K. Willett, and F. Hasegawa, "Near optimal multiuser detection in synchronous CDMA using probabilistic data association," IEEE Commun. Lett., vol. 5, no. 9, pp. 361-363, Sep. 2001.

[2] D. Pham, K. R. Pattipati, P. K. Willett, and J. Luo, "A generalized probabilistic data association detector for multiple antenna systems," IEEE Commun. Lett., vol. 8, no. 4, pp. 205-207, Apr. 2004.

[3] S. Liu and Z. Tian, "Near-optimum soft decision equalization for frequency selective MIMO channels," IEEE Trans. Signal Process., vol. 52, no. 3, pp. 721-733, Mar. 2004.

[4] S. Bavarian and J. K. Cavers, "A new framework for soft decision equalization in frequency selective MIMO channels," IEEE Trans. Commun., vol. 57, no. 2, pp. 415 -422, Feb. 2009.

[5] S. Yang, T. Lv, R. G. Maunder, and L. Hanzo, "Unified bit-based probabilistic data association aided MIMO detection for high-order QAM constellations," IEEE Trans. Veh. Technol., vol. 60, no. 3, pp. 981-991, Mar. 2011.

[6] - "Distributed probabilistic-data-association-based soft reception employing base station cooperation in MIMO-aided multiuser multicell systems," IEEE Trans. Veh. Technol., vol. 60, no. 7, pp. 3532-3538, Sep. 2011.

[7] S. Yang, L. Wang, T. Lv, and L. Hanzo, "Approximate Bayesian probabilistic-data-association-aided iterative detection for MIMO systems using arbitrary $M$-ary modulation," IEEE Trans. Veh. Technol., vol. 62, no. 3, pp. 1228-1240, Mar. 2013.

[8] S. Yang, T. Lv, R. G. Maunder, and L. Hanzo, "From nominal to true $a$ posteriori probabilities: an exact Bayesian theorem based probabilistic data association approach for iterative MIMO detection and decoding," IEEE Transactions on Communications, vol. 61, no. 7, pp. 2782-2793, Jul. 2013.

[9] F. D. Neeser and J. L. Massey, "Proper complex random processes with applications to information theory," IEEE Trans. Inf. Theory, vol. 39, no. 4, pp. 1293-1302, Jul. 1993.

[10] T. Adali, P. Schreier, and L. Scharf, "Complex-valued signal processing: The proper way to deal with impropriety," IEEE Trans. Signal Process., vol. 59, no. 11, pp. 5101-5125, Nov. 2011.

[11] P. M. Lee, Bayesian Statistics: An Introduction, 3rd ed. Chichester, UK: Wiley, 2004.

[12] B. M. Hochwald and S. ten Brink, "Achieving near-capacity on a multiple-antenna channel," IEEE Trans. Commun., vol. 51, no. 3, pp. 389-399, Mar. 2003.

[13] S. ten Brink, "Convergence behavior of iteratively decoded parallel concatenated codes," IEEE Trans. Commun., vol. 49, no. 10, pp. 1727 1737, Oct. 2001.

[14] C. Berrou and A. Glavieux, "Near optimum error correcting coding and decoding: turbo-codes," IEEE Trans. Commun., vol. 44, no. 10, pp. 1261-1271, Oct. 1996. 